\title{
Identification of microRNAs in blood and urine as tumour markers for the detection of urinary bladder cancer
}

\author{
ANGELIKA TÖLLE ${ }^{1}$, MONIKA JUNG ${ }^{1}$, SILKE RABENHORST $^{1}$, \\ ERGIN KILIC $^{2}$, KLAUS JUNG ${ }^{3}$ and STEFFEN WEIKERT ${ }^{1}$ \\ ${ }^{1}$ Department of Urology, Charité - Universitätsmedizin Berlin, 10117 Berlin; \\ ${ }^{2}$ Institute of Pathology, Charité - Universitätsmedizin Berlin, 10117 Berlin, \\ ${ }^{3}$ Berlin Institute for Urologic Research, 10117 Berlin, Germany
}

Received April 25, 2013; Accepted June 14, 2013

DOI: $10.3892 /$ or.2013.2621

\begin{abstract}
Since differential expression of microRNAs (miRNAs) has been found to be highly associated with several types of cancer, the goal of the present study was to identify an miRNA fingerprint as a non-invasive diagnostic tool to detect urinary bladder cancer using the easily accessible samples of whole blood and urine. Blood and urine samples from 4 controls and from patients suffering from superficial and invasive bladder cancer were analyzed using miRNA microarray consisting of 754 human miRNAs from the Sanger database v14. Using RT-qPCR technique, 6 of the differentially expressed miRNAs were validated in the controls (20 blood, 19 urine samples) and patients with superficial (18 blood, 16 urine samples) or invasive (20 blood and urine samples each) tumours. Three blood miRNAs (miR-26b-5p, miR-144-5p, miR-374-5p) were found to be significantly upregulated in invasive bladder tumour patients $(\mathrm{P}<0.05)$ when compared to the control group. The expression of 2 miRNAs (miR-618, miR-1255b-5p) in the urine of patients with invasive tumours was significantly $(\mathrm{P}<0.05)$ increased in comparison to the control group. Blood miR-26b-5p detected the presence of invasive bladder tumours with $94 \%$ specificity and $65 \%$ sensitivity. The urine miR-1255b-5p reached $68 \%$ specificity and $85 \%$ sensitivity in the diagnosis of invasive tumours. This pilot study represents the first characterization of an miRNA profile for urinary bladder tumours in whole blood samples. In addition, it was shown that invasive bladder tumours could be identified by differentially expressed urine miRNAs. Further studies are needed to test the clinical usefulness for bladder cancer detection and surveillance.
\end{abstract}

Correspondence to: Dr Angelika Tölle, Department of Urology, Charité - Universitätsmedizin Berlin, Charitéplatz 1, 10117 Berlin, Germany

E-mail: angelika.toelle@charite.de

Key words: microRNAs, bladder cancer, whole blood, urine, tumour marker, non-invasive diagnostic

\section{Introduction}

Urinary bladder cancer (BC) is the fifth most common malignancy. In the US, every year approximately 15,000 individuals succumb to this disease (1). The diagnostic 'gold standard' in cases of urinary BC is cystoscopy, an invasive and relatively expensive procedure. Non-invasive urine cytology shows diagnostic high specificity but has the disadvantage of low sensitivity. Numerous urine-based tests such as bladder tumour antigen (BTA), nuclear matrix protein 22 (NMP22) or FISH cannot replace cystoscopy or cytology for diagnosis and follow-up examination (2). Thus, the development of non-invasive diagnostic markers for the detection of $\mathrm{BC}$ is an essential objective in urology.

Several studies have shown that microRNAs (miRNAs) are involved in various biological processes, including tumourigenesis $(3,4)$. miRNAs act as oncogenes or tumour-suppressor genes depending on the target that they regulate. The detection of differentially expressed miRNAs in tissues and body fluids is becoming increasingly important. Specific miRNA profiles have been identified for several types of carcinomas, among them urinary $\mathrm{BC}$. The results are partly contradictory since different array platforms, sample processing modes, patient groups, and normalization strategies were used (5-11). miRNAs in easily accessible body fluids could reflect tissue situation and are therefore of great, diagnostic and prognostic interest. Data regarding the detection of miRNAs in urine samples have been rarely published (12-16). In bladder cancer, urinary miR-126 was described as a specific indicator of BC (16), while overexpressed miR-96 and miR-183 were found to be correlated to tumour stage and grade (15). Another study showed a characteristic panel of 3 miRNAs (miR-15b/ miR-135b/miR-1224-3p) in urine sediments for detecting urothelial cell carcinomas (12).

We analyzed the miRNA expression profile in whole blood and urine samples from patients with superficial and invasive bladder tumours in comparison with healthy individuals. In a first step, an miRNA microarray technology identified a panel of significant miRNAs. In a second step, following investigation of a greater number of patients, the expression level of a limited number of selected differentially expressed miRNAs was validated using real-time quantitative PCR (RT-qPCR). 
In blood samples, 3 miRNAs (miR-26b-5p, miR-144-5p, miR-374b-5p) were identified as discriminators between invasive tumours and the control group, while 3 other miRNAs (miR-520e, miR-618, miR-1255b-5p) were significantly increased in urine samples of patients with invasive tumours.

\section{Materials and methods}

Blood and urine of patient groups and controls. Blood and urine samples were collected from BC patients without metastasis and from a control group including healthy individuals and patients with benign urological diseases. All samples were collected immediately before transurethral resection of $\mathrm{BC}$ or cystectomy at the Charité University Hospital between 2010 and 2012. Following surgery, tumour classification was established according to the UICC 2010 TNM system. The study was approved by the Human Use Committee of our hospital (file: EA1/187/09), and informed consent of all patients was obtained. All BCs were transitional cell carcinomas; samples from patients with CIS were not investigated. Blood samples were collected and stored using PAXgene ${ }^{\mathrm{TM}}$ blood RNA tubes (Becton-Dickinson, Heidelberg, Germany). Urine collection $(30 \mathrm{ml})$ was performed with a urine RNA concentration, preservation and isolation kit (Norgen Biotek Corp., Thorold, Canada). The study was performed on two independent sample sets of blood and urine. Set one included 12 blood and urine (Table I) and set two, 58 blood and 55 urine samples (Table II), respectively. The demographic and clinicopathological characteristics of the study groups are summarized in Tables I and II. Samples from set one were used for miRNA profiling on a microarray platform and samples from set two were used for the RT-qPCR validation of miRNAs.

RNA isolation from blood and urine samples. Total RNA from the PAXgene ${ }^{\mathrm{TM}}$ blood samples was isolated using the PAXgene Blood miRNA kit (Qiagen, Hilden, Germany). The Norgen kit was used for the isolation of total RNA from urine samples including DNase treatment. RNA was collected in $300 \mu 1$ elution buffer and concentrated using the Concentrator 5301 (Eppendorf, Hamburg, Germany). The concentrated RNA was dissolved in a final volume of $70 \mu l$ of RNase-free water. As we detected DNA despite the DNase treatment step in the Norgen isolation procedure, we executed an additional DNase treatment using the Ambion ${ }^{\circledR}$ Turbo DNA-free ${ }^{\mathrm{TM}}$ kit (Applied Biosystems, Foster City, CA, USA). Quant-iT ${ }^{\mathrm{TM}}$ RiboGreen ${ }^{\circledR}$ RNA reagent (Molecular Probes, Göttingen, Germany) was applied for urine RNA quantification on the LightCycler 1.5 (17).

microRNA microarray experiments. Microarray experiments were carried out using TaqMan ${ }^{\circledR}$ array human microRNA cards (Applied Biosystems) including 754 human miRNAs from the Sanger database v14 and 8 control miRNAs. The urine RNA samples were investigated using an additional pre-amplification step. Reverse transcription was performed with $3 \mu \mathrm{l}$ blood RNA ( 200 $\mathrm{ng} / \mu \mathrm{l})$ or urine RNA $(\sim 10 \mathrm{ng} / \mu \mathrm{l})$. On the microarray cards, miRNAs were quantified until a Cq-threshold of 32. The expression level was calculated using the formula $2^{-\Delta \mathrm{Cq}}$ and normalized to snRNA U6. In this report, the current nomenclature of miBase v 19 was used.
Table I. Clinical and demographic characteristics of the patients in set one for the study of miRNAs in blood and urine samples using the TaqMan microarray platform.

\begin{tabular}{lcllc}
\hline Patient no. & Age (years) & Gender & \multicolumn{1}{c}{ Stage } & Grade \\
\hline 1 & 58 & Male & Healthy & - \\
2 & 39 & Female & Healthy & - \\
3 & 68 & Male & Healthy & - \\
4 & 56 & Female & Healthy & - \\
5 & 73 & Male & Superficial Ta & G1, low \\
6 & 61 & Male & Superficial Ta & G1, low \\
7 & 63 & Male & Superficial Ta & G2, high \\
8 & 63 & Male & Superficial Ta & G1, low \\
9 & 66 & Female & Invasive T2a & G3 \\
10 & 78 & Male & Invasive T2 & G3 \\
11 & 66 & Male & Invasive T3b & G3 \\
12 & 91 & Female & Invasive T1 & G3 \\
\hline
\end{tabular}

microRNA RT-qPCR. For each study group, 6 miRNAs were selected from the large number of differentially expressed miRNAs to validate them in blood and urine samples. The PCR reaction was carried out under the following conditions. Reverse transcription was performed using $6 \mathrm{ng}$ blood and $1 \mathrm{ng}$ urine RNA, respectively, and TaqMan ${ }^{\circledR}$ microRNA reverse transcription kit (Applied Biosystems). In the case of blood samples, the PCR reaction included $1 \mu \mathrm{l}$ miRNA-specific cDNA, 1X TaqMan ${ }^{\circledR}$ Universal PCR Master Mix and gene-specific 1X TaqMan ${ }^{\circledR}$ microRNA assay solution (Applied Biosystems). In the case of urine samples, a modification occurred, since $0.2 \mathrm{X}$ TaqMan ${ }^{\circledR}$ MicroRNA assay solution was used. Afterwards a pre-amplification reaction only for the urine samples was performed with $1 \mu \mathrm{l}$ cDNA, TaqMan ${ }^{\circledR}$ Preamp Mix and gene-specific 0.05X TaqMan ${ }^{\circledR}$ microRNA assay solution. Each PCR run included a non-template control with water instead of cDNA. An miRNA was detected with a Cq-value $\leq 35$ and was normalized to the expression of small RNA U6 for blood miRNAs. The urine miRNAs were normalized to $1 \mathrm{ng}$ urine RNA which was used for cDNA amplification. All PCR reactions with sample set one were carried out in triplicates.

All PCR reactions with the sample set two were carried out under the same conditions. All PCR reactions were carried out in duplicates. Standard curves of diluted miRNA-specific cDNA miR-26b-5p for blood and miR-29a-3p for urine as well as snRNA U6 from pooled normal bladder tissue was generated and used for calculation of expression values. The resulting expression values are provided as arbitrary units. The analytical reliability of PCR methods was characterized by the efficiency of amplification between 1.80 and 2.08 for snRNA U6 and miR-26b-5p, respectively. The precision of repeated measurements was tested with snRNA U6 $(n=10)$ and miR-26b-5p $(\mathrm{n}=8)$ of 0.47 and $0.63 \%$ at threshold cycles (Cq-values) of $27.3 \pm 0.13$ and $28.8 \pm 0.18$, respectively. These details related to the blood RNA-isolates.

Sample set two (Table II) was used for validation. The blood miRNAs were normalized to snRNA U6 and the urine 
Table II. Clinical and demographic characteristics of the patients in set two for the study of miRNAs in blood and urine samples using RT-qPCR.

Characteristics Control group Superficial Ta Invasive $>$ T1

\section{Blood samples}

Total no.

Age, in years

median (range) 43 (27-82)

$67(30-83)$

69 (46-76)

Gender

Male

Female

\section{5}

15

8

10

12

Grade

G1, low

G2, low

G2, high

G3

$$
8
$$

9

1

16

Total no.

Age, in years

median (range) 49 (27-82)

Gender

Male

4

Female

Grade

G1, low

G2, low

G2, high

G3

7

(

6

92

13

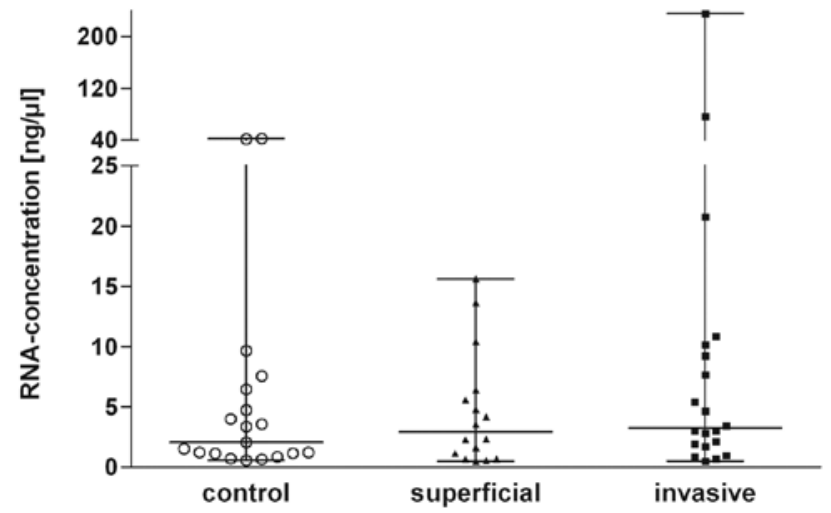

Figure 1. RNA concentration in urine RNA isolates after concentration and DNase treatment. The Kruskal-Wallis test showed no differences between the 3 groups $(\mathrm{P}=0.07)$.

miRNAs, microRNAs; RT-qPCR, real-time quantitative PCR.

miRNAs to RNA concentration of the urine RNA isolate after concentration and DNase-treatment. Afterwards, the median expression for miRNA in each group was computed and tested for significance between the groups.

Statistical analysis. Statistical analyses were performed with the software GraphPad Prism for Windows, version 5 (GraphPad Software, San Diego, CA, USA). Outliers in the control groups were identified with the Grubbs' test. The two outliers in the blood control group were from the same healthy individuals and were excluded from further analysis. The samples from these individuals were collected in 2010 and no bladder cancer was diagnosed at the end of 2012. The outliers in the urine control group were caused by different healthy individuals so that these values were not excluded from further analysis. The Kruskal-Wallis and Mann-Whitney U tests were used for data comparison between groups. Medians were considered significantly different at $\mathrm{P}<0.05$ (two tailed).

Receiver operation characteristic (ROC) analysis (MedCalc, version 12 for Windows, Mariakerke, Belgium) was used to characterize the capacity of a single miRNA or a combination of miRNAs to discriminate between urine samples from controls and bladder cancer patients.

\section{Results}

Characteristics of the patient groups and samples. Blood and urine samples of set one were collected from 4 controls and 4 patients with superficial and invasive bladder cancer, respectively (Table I). Sample set two included varying numbers of blood and urine samples of controls and patients with superficial tumours (Table II). The analyzed blood and urine samples were not always collected from the same patient due to the very low RNA concentration in some urine samples. The median age between $\mathrm{BC}$ patients and the control group was significantly different (67 vs. 43 years, $\mathrm{P}<0.05$ ) in blood and urine set two. To date, reports concerning age-dependent changes in miRNA expression in body fluids are not available. The snRNA U6 was undetectable in 13 of 55 urine RNA samples (23.6\%), and was therefore not used for normalization. A similar observation was reported by Hanke et al (16).

Identification of differentially expressed miRNAs with microarray experiments. The characteristics of the RNA samples are summarized in Table III. After all preparation steps, the urine RNA concentrations were comparable (Kruskal-Wallis; $\mathrm{P}=0.07$ ) in all 3 groups (Fig. 1). Several urine RNA isolates showed low RIN values $<3$. However, since the detection of miRNAs was not compromised in RNA preparations with a low RIN value (18) all preparations were used for further analysis. The microarray experiments identified $>100$ differentially expressed miRNAs in blood and $>80$ miRNAs in urine. In blood samples, only increased miRNAs were observed in comparison to the controls. The number of detected miRNAs in the tumour group was greater than that in the controls. In urine samples from invasive tumours, expression differences were observed for 60 miRNAs. A short list of differently expressed miRNAs in the samples are given in Tables IV and V. There was no similarity between miRNAs with the greatest expression differences between tumour and control in blood and in urine. Therefore, various miRNAs were selected for validation with RT-qPCR in blood and urine. miRNAs in the patient samples with an increased fold-change 
Table III. Characteristics of the blood and urine RNA samples.

\begin{tabular}{|c|c|c|c|}
\hline Characteristics & Control group & Superficial Ta & Invasive $\geq \mathrm{T} 1$ \\
\hline \multicolumn{4}{|l|}{ Blood samples } \\
\hline Total no. & 20 & 18 & 20 \\
\hline $\mathrm{A}_{260} / \mathrm{A}_{280}{ }^{\mathrm{a}}$ (arithmetic mean $\left.\pm \mathrm{SD}\right)$ & $2.02 \pm 0.04$ & $2.04 \pm 0.04$ & $2.03 \pm 0.04$ \\
\hline RNA integrity , value (range) & 7.2-9.4 & & $8.8-9.2$ \\
\hline Concentration $^{\mathrm{a}}(\mathrm{ng} / \mu \mathrm{l})($ arithmetic mean $\pm \mathrm{SD})$ & $222.7 \pm 143.6$ & $177.8 \pm 57.9$ & $218.7 \pm 162.4$ \\
\hline \multicolumn{4}{|l|}{ Urine samples } \\
\hline Total no. & 19 & 16 & 20 \\
\hline $\mathrm{A}_{260} / \mathrm{A}_{280}{ }^{\mathrm{a}}$ (arithmetic mean $\left.\pm \mathrm{SD}\right)$ & $1.40 \pm 0.52$ & $1.28 \pm 0.49$ & $1.70 \pm 0.56$ \\
\hline RNA integrity ${ }^{\mathrm{b}}$, value (range) & $1.7-2.9$ & & 2.4 \\
\hline Concentration $^{\mathrm{c}}(\mathrm{ng} / \mu \mathrm{l})(\operatorname{arithmetic}$ mean $\pm \mathrm{SD})$ & $7.08 \pm 12.58$ & $4.63 \pm 4.75$ & $20.06 \pm 53.33$ \\
\hline
\end{tabular}

${ }^{a}$ RNA content and quality were determined with NanoDrop ND 1000 spectrophotometer (Thermo Fischer Scientific, Wilmington, DE, USA); ${ }^{b}$ RNA quality was determined using Bioanalyzer (Agilent Technologies, Santa Clara, CA, USA); ${ }^{\circ}$ RA concentration was determined after all RNA-preparation steps using Quant $\mathrm{iT}^{\mathrm{TM}}$ RiboGreen ${ }^{\circledR}$ RNA reagent.

Table IV. Short list of differentially expressed miRNAs in blood samples (microarray results).

\begin{tabular}{|c|c|c|c|}
\hline \multirow[b]{2}{*}{ miRNA } & \multicolumn{3}{|c|}{ Fold changes } \\
\hline & $\begin{array}{l}\text { Superficial/ } \\
\text { control }\end{array}$ & $\begin{array}{l}\text { Invasive/ } \\
\text { control }\end{array}$ & $\begin{array}{l}\text { Tumour/ } \\
\text { control }\end{array}$ \\
\hline hsa-miR-625-3p & 10.97 & 17.80 & 13.33 \\
\hline hsa-miR-574-3p & 5.61 & 13.23 & 7.76 \\
\hline hsa-miR-361-5p & 0.67 & 8.86 & 7.04 \\
\hline hsa-miR-374b-5p & 2.44 & 7.93 & 4.99 \\
\hline hsa-miR-144-5p & 21.88 & 23.86 & 23.86 \\
\hline hsa-miR-671-3p & 2.93 & 7.13 & 5.44 \\
\hline hsa-miR-486-5p & 2.29 & 4.65 & 3.64 \\
\hline hsa-miR-26b-5p & 2.63 & 8.53 & 5.99 \\
\hline hsa-miR-374a-5p & 5.49 & 4.95 & 4.95 \\
\hline hsa-miR-485-3p & 1.84 & 7.96 & 3.48 \\
\hline hsa-miR-26a-5p & 2.29 & 6.82 & 4.29 \\
\hline hsa-miR-18a-3p & 3.13 & 5.53 & 3.82 \\
\hline hsa-miR-629-3p & 6.41 & 11.61 & 10.45 \\
\hline hsa-miR-550a-5p & 4.26 & 10.56 & 5.18 \\
\hline hsa-miR-484 & 2.43 & 3.87 & 3.46 \\
\hline hsa-miR-99b-5p & 2.55 & 6.61 & 4.52 \\
\hline hsa-miR-139-5p & 2.21 & 5.38 & 2.72 \\
\hline hsa-miR-342-3p & 2.87 & 5.61 & 4.07 \\
\hline hsa-miR-21-5p & 2.94 & 4.10 & 3.59 \\
\hline hsa-miR-192-5p & 3.64 & 4.60 & 3.77 \\
\hline
\end{tabular}

The 6 highlighted miRNAs in bold were selected for validation. miRNAs, microRNAs; hsa-miR, Homo sapiens miRNA.

$\geq 2.5$ or a decreased fold-change $\geq 10$ were selected for further investigation. In addition, miR-139-5p was also selected for further validation as it showed a characteristic differential expression between tumour and normal tissues $(6,7,10)$. Other
Table V. Short list of differentially expressed miRNAs in urine samples (microarray results).

\begin{tabular}{lrrr}
\hline & \multicolumn{3}{c}{ Fold changes } \\
\cline { 2 - 4 } miRNA & $\begin{array}{c}\text { Superficial/ } \\
\text { control }\end{array}$ & $\begin{array}{c}\text { Invasive/ } \\
\text { control }\end{array}$ & $\begin{array}{r}\text { Tumour/ } \\
\text { control }\end{array}$ \\
\hline hsa-miR-21-5p & 94.28 & -5.48 & 5.48 \\
hsa-miR-202-3p & 1.73 & 17.78 & 15.42 \\
hsa-miR-302a-3p & -3.17 & 18.18 & 9.78 \\
hsa-miR-483-5p & $\mathbf{- 4 . 3 2}$ & $\mathbf{4 . 5 2}$ & $\mathbf{3 . 5 8}$ \\
hsa-miR-548d-5p & 1.34 & 46.91 & 44.01 \\
hsa-miR-618 & $\mathbf{1 . 9 3}$ & $\mathbf{1 4 . 2 3}$ & $\mathbf{8 . 1 2}$ \\
hsa-miR-520e & $\mathbf{- 1 1 . 1 3}$ & $\mathbf{5 , 1 5 4 . 5 5}$ & $\mathbf{3 , 4 7 9 . 9 0}$ \\
hsa-miR-516-3p & 1.19 & 3.09 & 2.05 \\
hsa-miR-520c-3p & 2.70 & 6.90 & 4.89 \\
hsa-miR-1303 & 1.60 & 3.94 & 3.25 \\
hsa-miR-1255b-5p & $\mathbf{1 . 2 1}$ & $\mathbf{2 1 . 3 5}$ & $\mathbf{1 6 . 0 5}$ \\
hsa-miR-875-5p & $\mathbf{4 . 1 2}$ & $\mathbf{- 4 0 . 9 5}$ & $\mathbf{- 2 2 . 1 5}$ \\
hsa-miR 29a-3p & $\mathbf{- 1 0 . 1 1}$ & $\mathbf{- 2 6 , 0 1 3}$ & $\mathbf{- 2 , 5 3 5 . 4}$ \\
hsa-miR-222-3p & -5.42 & -38.08 & -29.24 \\
hsa-miR-342-3p & -23.42 & -95.82 & -62.06 \\
hsa-let-7e-5p & -88.28 & -114.43 & -103.31 \\
\hline
\end{tabular}

The 6 highlighted miRNAs in bold were selected for validation. miRNAs, microRNAs; hsa-miR, Homo sapiens miRNA.

miRNAs with expression alterations in tumour tissues such as miR-21 and miR-205 $(19,20)$ were not included in the validation set of miRNAs since they were not detected in the blood or urine samples.

Validation of differentially expressed miRNAs with RT-qPCR. The quantification of blood miRNAs in sample set 2 showed an increased expression ( $\geq 2.0$-fold) in invasive tumours 
Table VI. Receiver operating characteristic curves are shown for 6 microRNAs detected in blood and urine samples from 20 patients with invasive bladder carcinoma in comparison with 20 and 19 healthy individuals, respectively.

\begin{tabular}{llllcc}
\hline miRNA & AUC & $95 \%$ CI & Cut-off $^{\text {a }}$ & Sensitivity (\%) & Specificity (\%) \\
\hline $\begin{array}{l}\text { Blood samples } \\
\text { hsa-miR-26b-5p }\end{array}$ & 0.824 & $0.663-0.929$ & 0.59 & & \\
hsa-miR-144 5p & 0.779 & $0.613-0.899$ & 0.52 & 70.0 & 94.1 \\
hsa-miR-374-5p & 0.774 & $0.606-0.894$ & 0.54 & 60.0 & 82.4 \\
$\begin{array}{l}\text { Urine samples } \\
\text { hsa-miR-520e }\end{array}$ & 0.679 & $0.510-0.819$ & 0.44 & 70.0 & 63.1 \\
hsa-miR-618 & 0.692 & $0.524-0.830$ & 4.4 & 70.0 & 68.4 \\
has-miR-1255b-5p & 0.764 & $0.601-0.885$ & 5.32 & 85.0 & 68.4 \\
\hline
\end{tabular}

microRNAs, miRNAs; hsa-miR, Homo sapiens miRNA; acut off corresponding with highest Youden index.

A

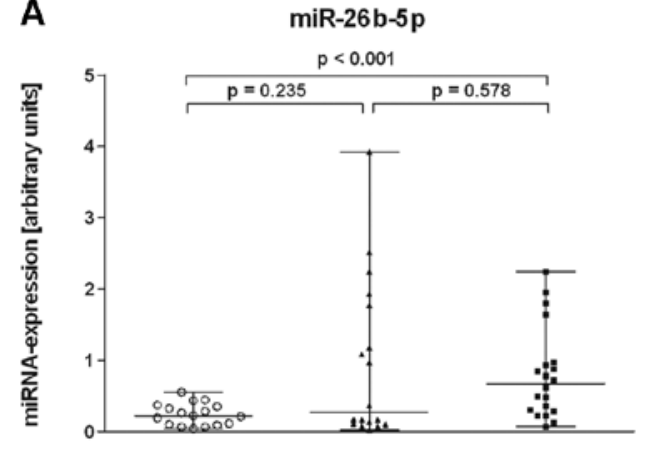

C

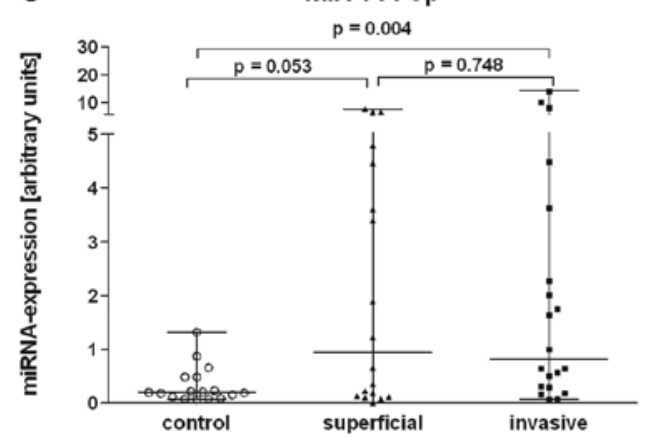

B

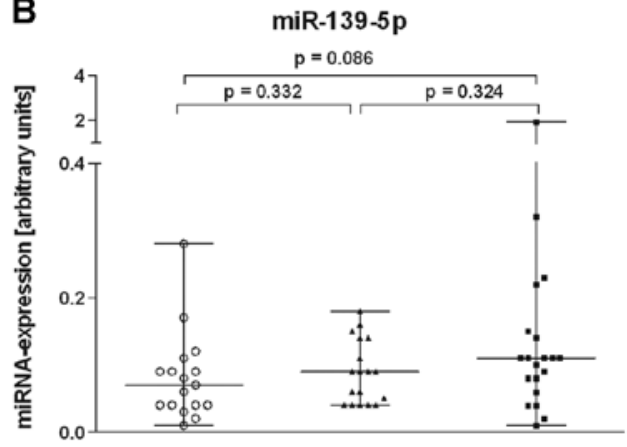

DiR-374b-5p

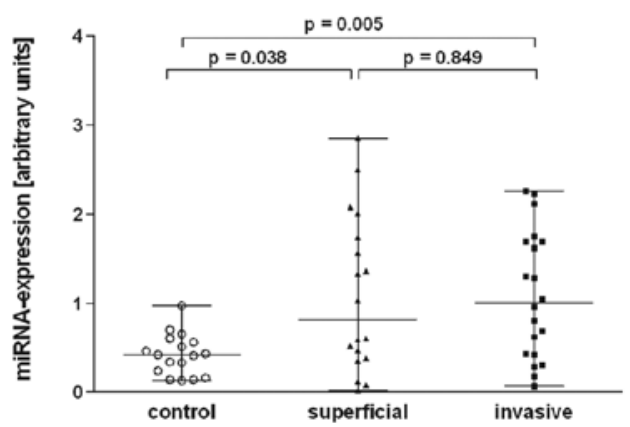

Figure 2. Expression levels of 4 miRNAs in blood samples from bladder tumour patients and controls. RT-qPCR analyses were performed with 20 controls, 18 superficial and 20 invasive tumours. The expression values were calculated using a relative standard curve. The relative miRNA expression was normalized to the amount of snRNA U6. Statistical significance was determined by Mann-Whitney U test. (A) miR-26b-5p was significantly differentially expressed between control and invasive tumours. (B) miR-139-5p was not significantly expressed among all groups. (C) miR-144-5p was significantly differentially expressed between control and invasive tumours. (D) miR-374-5p was significantly differentially expressed between control and superficial tumours and between control and invasive tumours.

for miR-26b-5p, miR-144-5p and miR-374b-5p (Fig. 2) in comparison to the controls. The other 3 miRNAs (miR-139-5p, miR-574-3p, miR-625-3p) were only $>1.5$ times increased. The expression difference for these miRNAs observed in microarray experiments could not be reproduced in the RT-qPCR analysis. The expression of miRNAs miR-26b-5p $(\mathrm{P}<0.001)$, miR-144-5p $(\mathrm{P}=0.004)$ and $\mathrm{miR}-374 \mathrm{~b}-5 \mathrm{p}(\mathrm{P}=0.005)$ in blood of patients with invasive tumours was significantly increased in comparison to the control group. Only miR-374b-5p discriminated between control and patients with superficial tumours
$(\mathrm{P}=0.038)$. Expression differences of the other 2 miRNAs (miR-26-5p, miR-144p) between patients with invasive and superficial tumours were not significant (Fig. 2). When the blood samples from the cancer patients were compared as one group (invasive and superficial tumours together) with the control group, the expression differences were significant for all 3 miRNAs (miR-26b-5p, $\mathrm{P}=0.008$; miR-144-5p, $\mathrm{P}=0.005$ and $\mathrm{miR}-374 \mathrm{~b}-5 \mathrm{p}, \mathrm{P}=0.005)$.

The validation of urine miRNAs in sample set two showed an increasing expression ( $\geq 2.0$-fold) in invasive tumours for 
A
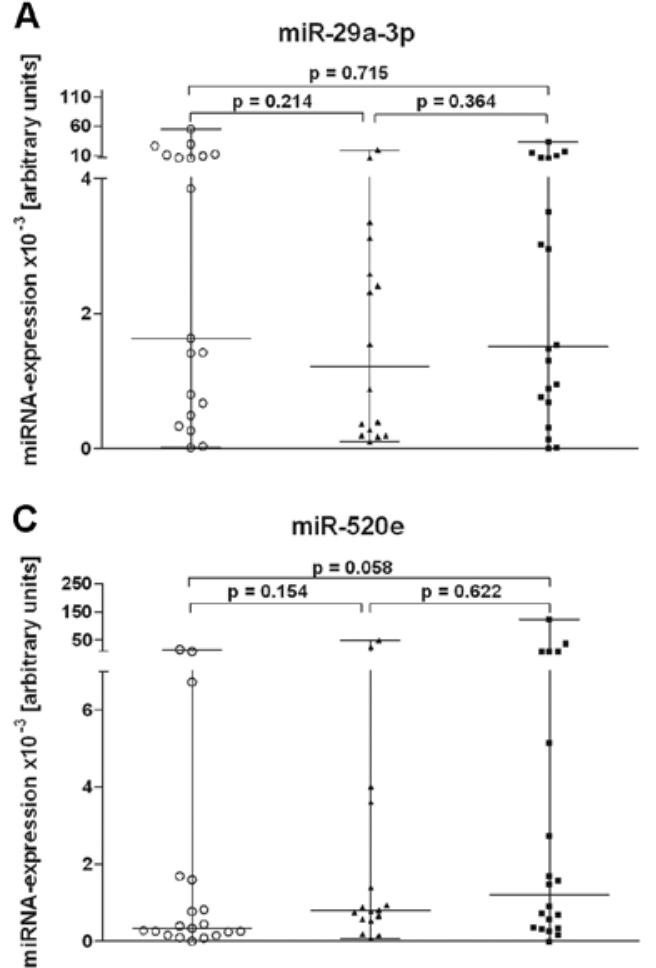

E

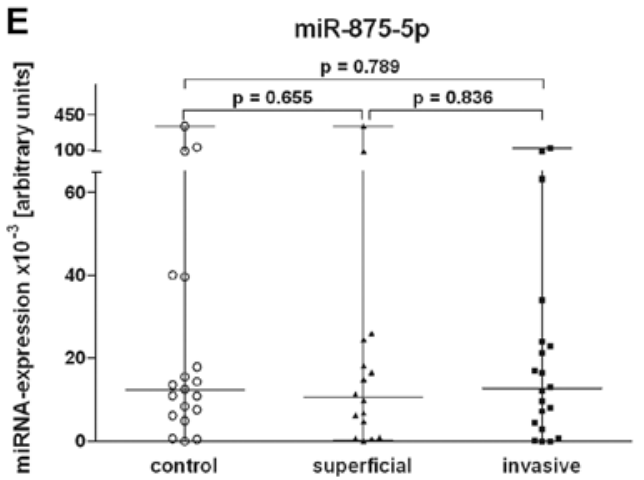

B

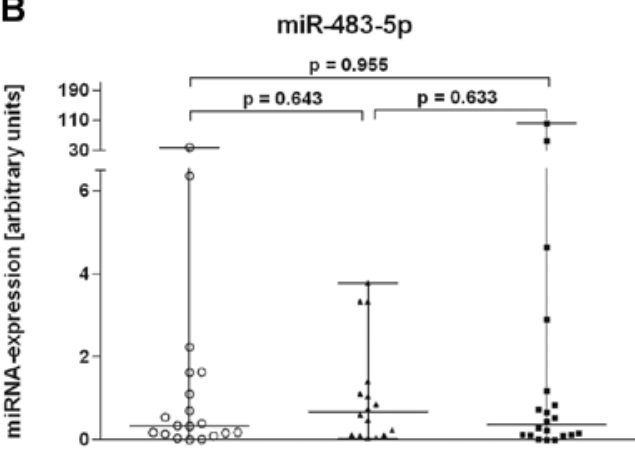

D

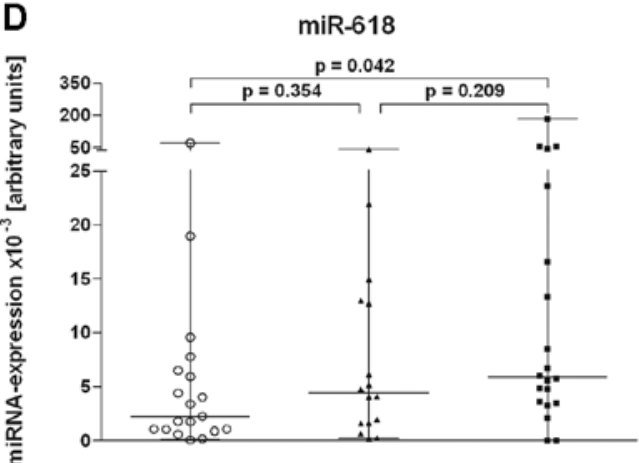

$\mathbf{F}$

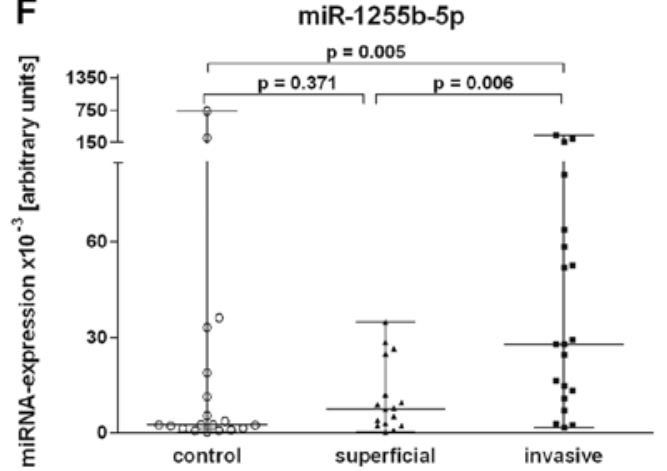

Figure 3. Expression levels of 6 miRNAs in urine samples from bladder tumour patients and controls. RT-qPCR analyses were performed with 19 controls, 16 superficial and 20 invasive tumours. The expression values were calculated using a relative standard curve. The relative miRNA expression was normalized with the RNA concentration of the urine RNA isolate after concentration and DNase treatment. Statistical significance was determined by Mann-Whitney U test. (A, B and E) miR-29a-3p, miR-483-5p and miR-875-5p were not significantly expressed among all groups. (C) miR-520e showed a tendency for higher expression in patients with invasive tumours when compared to the controls. (D and F) miR-618 and miR-1255b-5p were significantly differentially expressed between the controls and patients with invasive tumours, and miR-1255B-5p showed a significantly differentially expression between patients with superficial and invasive tumours.

miR-520e, miR-618 and miR-1255b-5p. The expression of urine miRNAs miR-618 $(\mathrm{P}=0.042)$ and miR-1255b-5p $(\mathrm{P}=0.005)$ in invasive tumours was significantly increased in comparison to the control group. The expression of miR-520e showed a tendency for increased values $(\mathrm{P}=0.058)$ in the urine of the invasive tumour group compared with the controls. Significant expression differences of these miRNAs between invasive and superficial tumours were not observed (Fig. 3). When the miRNAs of the urine samples from the entire tumour group were compared with those of the healthy controls, the expression differences were significant for miR-1255b-5p $(\mathrm{P}=0.024)$.

Receiver operation characteristic (ROC) analysis. ROC curve analysis demonstrated that each blood miRNA had a good specificity but low sensitivity for the identification of invasive tumours. All 3 urine miRNAs attained a sensitivity $\geq 70 \%$, but the specificity was equally low for the identification of invasive tumours (Table VI).

\section{Discussion}

miRNAs in blood and urine are of great interest from the diagnostic and prognostic point of view since they can be obtained by non-invasive or minimally invasive techniques. This is the first study concerning dysregulated miRNAs in whole blood samples from bladder cancer patients. From the 6 selected miRNAs that were found with increased expression in blood, 4 miRNAs (miR-26b-5p, miR-139-5p, miR-374b-5p, miR-574-3p) $(6,7,10,11)$ were also identified as markers in bladder cancer tissue. We confirmed increased miR-26b-5p 
and miR-374b-5p values in blood samples that reflected the increased values already described in bladder cancer tissue samples $(7,11)$. miR-26b-5p and miR-374-5p in blood showed both a specificity of $94 \%$ and sensitivities of 65 and $60 \%$, respectively, in detecting invasive tumours. Levels of the other 2 miRNAs miR-139-5p and miR-574-3p were unchanged in blood whereas their expression was found reduced in tumour tissue $(6,7,10)$. Additionally, this study identified miR-144-5p in blood samples as a new marker of invasive bladder tumours. There are no reports available concerning miR-144-5p in bladder cancer patients. Expression alterations for the 3 miRNAs with increased expression found in the blood samples in this study were reported in connection with other carcinomas. Lung cancer showed alterations in miR-26b and miR-374 (21,22). Changes in miR-144 expression levels were observed in hepatocellular, lung and prostate cancer tissues (23).

The investigation of miRNAs in whole blood samples includes miRNAs from blood cells, released tumour cells, exosomes as well as all circulating, cell-free miRNAs released from the tumour. Since the proportion of circulating miRNAs of the total miRNAs in blood samples is small, variations are mainly caused by the blood cells and may be the result of systemic reactions linked to the tumour disease. In conclusion, a tumour characteristic miRNA pattern results from a specific reaction of the organism $(24,25)$.

The second part of the present study referred to the analysis of the RNA profile in urine. Reports on bladder cancer-specific miRNA signatures in urine samples are rare $(12,15,16,26-28)$. Using the RT-qPCR-based microarray we investigated the abundance of 754 different human miRNA species. This is the most comprehensive examination of miRNA markers in urine after the report of Hanke et al (16) who tested 157 different human miRNAs. In several other studies $(15,26,27)$, miRNAs were selected as urinary markers based on the distinct expression differences found in tumour tissue. Other the authors investigated only urine sediment $(12,28)$. However, most of the above analyzed miRNAs in those studies were not detectable in the present microarray analysis of urine specimens. miR-192 and miR-222 (28), although they were detected in all urine samples, were not selected for further investigations as the Cq-differences between samples from patients and controls were $<2$.

In urine of the $\mathrm{BC}$ patients, using the microarray approach, we identified the 2 miRNAs miR-29a-3p and miR-483-5p out of the 6 selected miRNAs that had previously been described as characteristic differentially expressed markers in bladder cancer tissue $(7,9)$. However, these characteristics found in the initial sample set one could not be confirmed in sample set two. Still, the expression differences for miR-618 and miR-1255b-5p could be confirmed in the larger patient set two. For these 2 miRNAs, downregulated urinary excretion data has been described in hepatocellular and renal carcinoma $(29,30)$. Both miRNAs may be linked with carcinomas but the origin of their occurrence in urine is unclear. The expression of miR-520e showed a tendency for increased values in the urine of the invasive tumour group compared with the controls. The findings of Zhang et al (31) suggest that miR-520e may be a tumour-suppressor miRNA in hepatocellular carcinomas since $\mathrm{NF}-\kappa \mathrm{B}$-inducing kinase is one of the direct target genes of miR-520e. Further studies with a large sample size will confirm this tendency.

Commonly, small RNAs are used as endogenous controls to normalize the expression data of miRNAs in tissues. Our results showed that these endogenous controls are not suitable for normalization of urine data as snRNA U6 was not expressed equally in all samples. A systematic evaluation of normalization approaches including the use of potential reference genes for the analysis of urine miRNA has been lacking to date. For this reason, we normalized the miRNA excretion using the same quantity of total RNA for all measurements. This approach to normalization by using total RNA was suggested by Bustin (32) when reliable reference genes are not available. However, this approach requires a reliable quantification of RNA, which we achieved, despite the low RNA concentrations, using the fluorimetric RNA assay with RiboGreen ${ }^{\circledR}(17)$.

In conclusion, this pilot study discovered three dysregulated miRNAs in whole blood and 2 others in urine for the identification of bladder cancer patients. miR-26b-5p is significantly increased in the blood of invasive bladder cancer patients and increased miR-1255b-5p in urine samples is also characteristic. The receiver operating characteristic curve analyses indicated that blood miR-26b-5p alone (AUC $=0.824)$ and urine miR-1255b-5p alone (AUC $=0.764)$ provide significant accuracy for invasive bladder cancer diagnosis. This study serves as a basis for further studies to specify useful blood and urine miRNA signatures and their application for initial bladder cancer detection and surveillance.

\section{Acknowledgements}

This study was supported by grants from the European Regional Development Fund (EFRE), ProFIT grant no. 10146784. We are grateful to Sabine Weickmann for the excellent technical assistance.

\section{References}

1. Siegel R, Naishadham D and Jemal A: Cancer statistics, 2012. CA Cancer J Clin 62: 10-29, 2012.

2. Hakenberg OW: Epidemiologie, diagnose und urinbasierte untersuchungsverfahren beim harnblasenkarzinom. Onkologe 13: 1067-1079, 2007 (In German).

3. Calin GA and Croce CM: MicroRNA signatures in human cancers. Nat Rev Cancer 6: 857-866, 2006.

4. Bartel DP: MicroRNAs: genomics, biogenesis, mechanism, and function. Cell 116: 281-297, 2004.

5. Han Y, Chen J, Zhao X, et al: MicroRNA expression signatures of bladder cancer revealed by deep sequencing. PLoS One 6: e18286, 2011.

6. Yoshino H, Chiyomaru T, Enokida H, et al: The tumoursuppressive function of miR-1 and miR-133a targeting TAGLN2 in bladder cancer. Br J Cancer 104: 808-818, 2011.

7. Song T, Xia W, Shao N, et al: Differential miRNA expression profiles in bladder urothelial carcinomas. Asian Pac J Cancer Prev 11: 905-911, 2010.

8. Wang $\mathrm{G}$, Zhang $\mathrm{H}, \mathrm{He} \mathrm{H}$, et al: Up-regulation of microRNA in bladder tumor tissue is not common. Int Urol Nephrol 42: 95-102, 2010.

9. Dyrskjøt L, Ostenfeld MS, Bramsen JB, et al: Genomic profiling of microRNAs in bladder cancer: miR-129 is associated with poor outcome and promotes cell death in vitro. Cancer Res 69: 4851-4860, 2009.

10. Ichimi T, Enokida H, Okuno Y, et al: Identification of novel microRNA targets based on microRNA signatures in bladder cancer. Int J Cancer 125: 345-352, 2009.

11. Gottardo F, Liu CG, Ferracin M, et al: Micro-RNA profiling in kidney and bladder cancers. Urol Oncol 25: 387-392, 2007. 
12. Miah S, Dudziec E, Drayton RM, et al: An evaluation of urinary microRNA reveals a high sensitivity for bladder cancer. Br J Cancer 107: 123-128, 2012.

13. Wang G, Kwan BC, Lai FM, Chow KM, Li PK and Szeto CC: Elevated levels of miR-146a and miR-155 in kidney biopsy and urine from patients with IgA nephropathy. Dis Markers 30: 171-179, 2011.

14. Lorenzen JM, Volkmann I, Fiedler J, et al: Urinary miR-210 as a mediator of acute T-cell mediated rejection in renal allograft recipients. Am J Transplant 11: 2221-2227, 2011

15. Yamada Y, Enokida H, Kojima S, et al: MiR-96 and miR-183 detection in urine serve as potential tumor markers of urothelial carcinoma: correlation with stage and grade, and comparison with urinary cytology. Cancer Sci 102: 522-529, 2011.

16. Hanke M, Hoefig K, Merz $\mathrm{H}$, et al: A robust methodology to study urine microRNA as tumor marker: microRNA-126 and microRNA-182 are related to urinary bladder cancer. Urol Oncol 28: 655-661, 2010.

17. Cheong A, Fountain SJ and Beech DJ: Quantitative RT-PCR methods for investigation of low copy potassium channel gene expression in native murine arteries. Methods Mol Biol 491: 19-33, 2008.

18. Jung M, Mollenkopf HJ, Grimm C, et al: MicroRNA profiling of clear cell renal cell cancer identifies a robust signature to define renal malignancy. J Cell Mol Med 13: 3918-3928, 2009.

19. Neely LA, Rieger-Christ KM, Neto BS, et al: A microRNA expression ratio defining the invasive phenotype in bladder tumors. Urol Oncol 28: 39-48, 2010.

20. Catto JW, Miah S, Owen HC, et al: Distinct microRNA alterations characterize high- and low-grade bladder cancer. Cancer Res 69: 8472-8481, 2009 .

21. Gao W, Shen H, Liu L, Xu J, Xu J and Shu Y: MiR-21 overexpression in human primary squamous cell lung carcinoma is associated with poor patient prognosis. J Cancer Res Clin Oncol 137: 557-566, 2011.

22. Miko E, Czimmerer Z, Csánky E, Boros G, Buslig J, Dezso B and Scholtz B: Differentially expressed microRNAs in small cell lung cancer. Exp Lung Res 35: 646-664, 2009.
23. Wang W, Peng B, Wang D, Ma X, Jiang D, Zhao J and Yu L: Human tumor microRNA signatures derived from large-scale oligonucleotide microarray datasets. Int J Cancer 129: 1624-1634, 2011.

24. Kossenkov AV, Vachani A, Chang C, et al: Resection of non-small cell lung cancers reverses tumor-induced gene expression changes in the peripheral immune system. Clin Cancer Res 17: 5867-5877, 2011

25. Keller A, Leidinger $\mathrm{P}$, Borries A, et al: miRNAs in lung cancer - studying complex fingerprints in patient's blood cells by microarray experiments. BMC Cancer 9: 353, 2009.

26. Puerta-Gil P, García-Baquero R, Jia AY, et al: miR-143, miR-222, and miR-452 are useful as tumor stratification and noninvasive diagnostic biomarkers for bladder cancer. Am J Pathol 180: 1808-1815, 2012

27. Snowdon J, Boag S, Feilotter H, Izard J and Siemens DR: A pilot study of urinary microRNA as a biomarker for urothelial cancer. Can Urol Assoc J: 1-5, 2012.

28. Wang G, Chan ES, Kwan BC, Li PK, Yip SK, Szeto CC and $\mathrm{Ng} \mathrm{CF}$ : Expression of microRNAs in the urine of patients with bladder cancer. Clin Genitourin Cancer 10: 106-113, 2012.

29. Abdalla MA and Haj-Ahmad Y: Promising candidate urinary microRNA biomarkers for the early detection of hepatocellular carcinoma among high-risk Hepatitis C virus Egyptian patients. J Cancer 3: 19-31, 2012.

30. Hidaka H, Seki N, Yoshino H, et al: Tumor suppressive microRNA-1285 regulates novel molecular targets: Aberrant expression and functional significance in renal cell carcinoma. Oncotarget 3: 44-57, 2012.

31. Zhang S, Shan C, Kong G, Du Y, Ye L and Zhang X: MicroRNA$520 \mathrm{e}$ suppresses growth of hepatoma cells by targeting the NF- $\kappa \mathrm{B}$-inducing kinase (NIK). Oncogene 31: 3607-3620, 2012.

32. Bustin SA: Quantification of mRNA using real-time reverse transcription PCR (RT-PCR): trends and problems. J Mol Endocrinol 29: 23-39, 2002. 